\title{
A literature review on multi-echelon inventory management: the case of pharmaceutical supply chain
}

\author{
Nouçaiba Sbai ${ }^{1, \mathrm{a}}$, Abdelaziz Berrado ${ }^{1}$ \\ ${ }^{1}$ Equipe AMIPS-Ecole Mohammadia d'Ingénieurs, Université Mohammed 5, Rabat, Maroc
}

\begin{abstract}
Inventory management remains a key challenge in supply chain management. Many companies recognize the benefits of a good inventory management system. An effective inventory management helps reaching a high customer service level while dealing with demand variability. In a complex supply chain network where inventories are found across the entire system as raw materials or finished products, the need for an integrated approach for managing inventory had become crucial. Modelling the system as a multi-echelon inventory system allows to consider all the factors related to inventory optimization. On the other hand, the high criticality of the pharmaceutical products makes the need for a sophisticated supply chain inventory management essential. The implementation of the multi-echelon inventory management in such supply chains helps keeping the stock of pharmaceutical products available at the different installations. This paper provides an insight into the multi-echelon inventory management problem, especially in the pharmaceutical supply chain. A classification of several multi-echelon inventory systems according to a set of criteria is provided. A synthesis of multiple multi-echelon pharmaceutical supply chain problems is elaborated.
\end{abstract}

\section{Introduction}

Controlling the material flow from suppliers of raw material to final customers has always been a critical problem. Top management of all organizations in any sector of the economy have recognized the strategic importance of supply chain management [1]. Viewing the supply chain as a whole, adopting a strategic orientation with cooperative efforts and having a customer focus have always been the main characteristics of the supply chain management [2].

Innovation leaders focused on effective inventory management as an essential part of supply chain management. In fact, it leads to enable shorter lead times to customers, makes working capital available for sustained profitability and helps dealing with demand variability.

The complexity of supply chains nowadays and the high interaction between all its nodes, make the determination of optimal inventory policy so difficult [3]. Adopting local optimization in each site of the supply chain cannot always have good results. In fact, singleechelon inventory management assumes that inventory is located in only one storage point where demand is met and replenishments are added. Actually, inventory is located in many storage points that belong to the same system which made researchers think about integrated approaches and modeling the system as a multi-echelon inventory system [4].

The high responsibility of the multi-echelon pharmaceutical supply chain to ensure the delivery of medicine in the right conditions makes the inventory management of this system more complex [5].

In this paper, we focus our attention on multi-echelon inventory systems. We consider specifically the multiechelon inventory management in the pharmaceutical supply chain.

This paper is organized in three sections. The next section presents the multi-echelon inventory system. Then, we present in Section 3 a literature review on multi-echelon inventory management and especially in the pharmaceutical supply chain.

\section{Multi-echelon inventory system}

The multi-echelon supply chain has multiple stages and many players at each stage [6]. The products are first sent to a central warehouse then shipped to the distribution centers that make the delivery to the final customer.

A multi-echelon system is a network composed of stages that are grouped into echelons. Stages can represent physical locations, items in stock or in processing activities [7]. Figure 1 presents a clear illustration of a multi-echelon inventory system.

The echelon inventory is defined as the inventory between a stage in the supply chain and the final customer. That means, the inventory position added to all downstream installations inventory [8].

\footnotetext{
${ }^{a}$ Corresponding author: noucaibasbai@ research.emi.ac.ma
} 


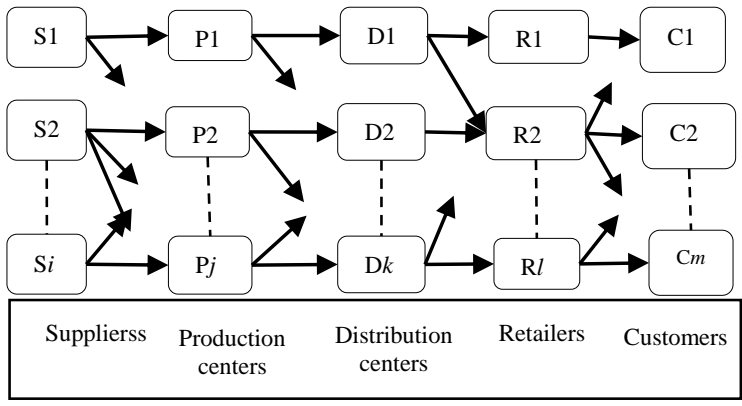

Fig. 1. An illustration of a multi-echelon supply chain with $i$ suppliers and $m$ customers.

\subsection{Structures of multi-echelon inventory system}

A multi-echelon inventory system may involve multiple suppliers and multiple customers for each inventory location in the system. On the basis of their network structure, multi-echelon inventory systems can be classified into serial systems, assembly systems, distribution systems and general systems.

In serial systems illustrated in Figure 2, each inventory location has at most one predecessor and one successor. In general, dealing with serial systems could be easier than with other types of multi-echelon systems.

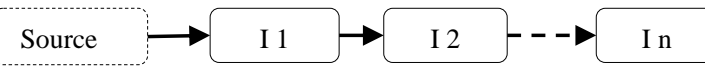

Fig. 2. Serial System of $n$ inventory locations

The second type is assembly systems. As illustrated in Figure 3, each installation has one immediate successor but multiple components can be assembled into a single item or final product which can result in many suppliers for a stock.

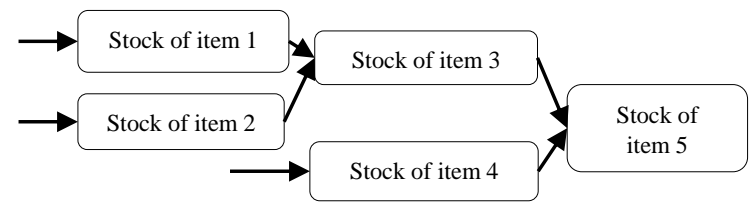

Fig. 3. Example of assembly system

A third structure is the distribution system in Figure4. This type is characterized by the increase of the number of parallel installations along the material flow, and this because a supplier may have multiple customers.

Finally, the general structure presented in Figure 5, can include any of the previous three structures as parts of the whole system.

\subsection{Characteristics of multi-echelon inventory system}

A multi-echelon inventory system has many characteristics that differentiate it from other systems and make it present many challenges in supply chain management. On the one hand, a multi-echelon inventory system is composed of multiple echelons that contain multiple stocks with physical locations.

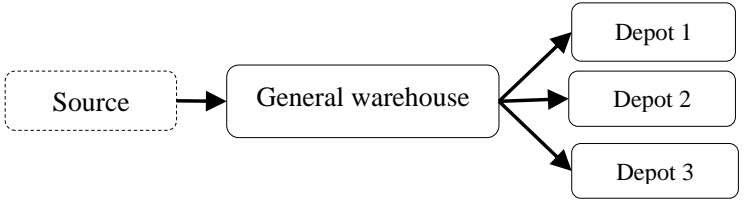

Fig. 4. Distribution system [4]

Each stock at a downstream position is a customer of one or multiple stocks at an upstream position [9]. On the other hand, this system can be characterized by the higher levels of average inventory which lead to have some trade-offs like having lower transportation costs, lower pipeline inventory, and faster response in meeting the demand at the end-customer level [4].

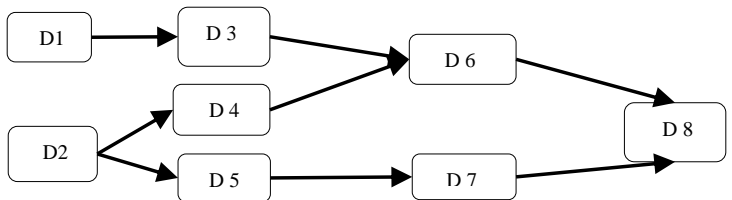

Fig. 5. Illustration of a general multi-echelon inventory system of 8 depots [1]

\subsection{Multi-echelon inventory management}

Inventory management approach has a direct impact on the overall inventory levels in the supply chain. Many companies used to manage their inventories in isolation or independently. Adopting a single-stage optimization can lead to have the sizing of inventories by applying the appropriate inventory models but it doesn't take into account the interdependencies between the different stages. Thus, multiple actors in the supply chain started to pay more attention to multi-echelon inventory management as a global optimization approach [4].

Multi-echelon inventory management or "the basic multi-activity inventory control problem", is establishing rules or policies which cause flows of the product through the network and which satisfy a prescribed performance objective [7]. As information is becoming centrally available for many companies, a more effective and efficient handling of inventories is possible. Multiechelon inventory management as an integrated approach, considers completely the factors related to inventory optimization: cost, variability, service and complexity. It sizes correctly the stock across the entire supply chain while taking into account the complex interdependencies between stages as well as variables that cause excess of inventory.

\section{Literature review}

In this section, we present a review on the models proposed for the optimization of multi-echelon inventory systems in general with a focus on pharmaceutical supply chain. We also define the problem of selecting the appropriate multi-echelon inventory system. 


\subsection{Literature review on multi-echelon inventory management}

The multi-echelon inventory system was discussed first by Clark and Scarf [10]. Before this research work, all papers treated the inventory problem by determining the optimal purchasing quantities at a single installation only. This paper presented the problem as a multi-installation model. Since that time, many authors tried to develop new approaches and ideas based on the model given by Clark and Scarf $[3,11,12,13,14,15,19]$.

In Table 1, we classified several models that were formulated by a lot of researchers in order to solve the multi-echelon inventory problem. Based on many assumptions and aspects considered in the single-stage optimization as well as those mentioned in the papers as characteristics of the models, we chose the following criteria as the ones that were assumed by the authors as necessary features in their formulations:

- Demand type: Deterministic or stochastic, static or non-static demand and the capability of the system to offer the option of emergency deliveries. In a deterministic model, external demands at each installation of the system are known with certainty. In a stochastic model, the demands obey a given probability distribution. For the case of static demand, parameters used to define external demand don't vary over time.

-Inventory/ Review policy: There are two replenishment policies often used in practice. The continuous review policy and the periodic review policy. In a continuous review model, the inventory position of the system is reviewed continuously over time and a quantity $\mathrm{Q}$ is ordered when the level of inventory reach the reorder point. In a periodic review model, the inventory status is reviewed at regular periodic intervals and the inventory level is raised to a predefined point. Besides that, this criteria includes the replenishment decisions (centralized or decentralized). In the centralized case, the objective is to find the optimal cost for the whole system. In the decentralized case, every installation in the system intends to optimize its own costs independently.

-Product: This criteria helps to classify the models into systems that are manufacturing a single product and those producing multiple products.

-Number of echelons: The number of echelons contained in the system.

\subsection{The case of pharmaceutical supply chain}

In the past, pharmaceutical supply chains were considered as a tool to deliver products to markets with taking into account security of supply. Recently, pharmaceutical industries are looking for new approaches in order to create additional benefits. There are a lot of actors in the pharmaceutical supply chain: primary manufacturing installations, distribution centers, wholesalers, hospitals and many others. The interdependencies between those components and the sensitivity of the pharmaceutical products make the call for sophisticated supply chain optimization techniques necessary.
The multi-echelon inventory problem in pharmaceutical supply chains was investigated by many authors. Tamil [5] treated a two-echelon supply chain inventory model for multiple pharmaceutical products under realistic problems in healthcare industries. He used a continuous review integrated production-distribution inventory model for a pharmaceutical company and a hospital supply chain , considering multiple pharmaceutical products, variable lead time and many constraints related to space availability and customer service level. Guerrero [15] presented a methodology to find near-optimal joint inventory control policies for a real case of a one-warehouse, n-retailer distribution system of infusion solutions at a University Medical Center in France. They considered emergency replenishments and target service level constraints under stochastic demand. The objective of the model was to optimize the inventory policy of the supply chain including the special constraints and operational differences that the healthcare industry demands. Kim [17] designed and developed an integrated supply chain management system for optimizing inventory control and reducing material handling costs of pharmaceutical products in healthcare sector. The supply chain studied was composed of pharmaceutical companies, a wholesaler and hospitals. The real time information sharing providing by the SCM system proposed helped forecasting demand more accurately by the wholesaler and reduce the total supply chain cost of pharmaceutical products by more than $30 \%$. Noorfa and Andrew [18] evaluated inventory management in the private healthcare sector in Malaysia. The paper focused on the inventory and delivery management process. After investigating a leading healthcare company in Malaysia who owns a wholesaler and a chain of medical clinics, the authors found two issues related to urgent orders due to poor inventory control methods and stock availability at the wholesaler problems. In order to reduce the operating cost and increase the customer service level, they suggested implementing a VMI approach based on the organization's current inventory management [18].

\subsection{Definition of the problem of selecting the appropriate multi-echelon inventory system}

The main problems faced by pharmaceutical supply chain sector are the need to balance future capacity with anticipated demand at the designed stage, and the difficulty to ensure responsiveness at the operation stage [16]. Nowadays, adopting supply chain management practices by applying techniques and methods developed in the industrial settings takes an important consideration by many health care organizations. In this context, our objective is to develop an approach to help decision makers choose the appropriate multi-echelon inventory system that satisfies the needs of the system and corresponds to their priorities.

\section{Conclusion}


In this paper, we presented a literature review on multiechelon inventory problem. Depending on the network structure, the multi-echelon inventory system may

Table 1.Classification of multi-echelon inventory models according to criteria used in the literature

\begin{tabular}{|c|c|c|c|c|}
\hline \multicolumn{2}{|c|}{ Network structure/ Criteria } & $\begin{array}{c}\text { Serial } \\
\text { system }\end{array}$ & Assembly system & Distribution system \\
\hline \multirow{3}{*}{ Demand } & Stochastic & {$[10]$} & & {$[11],[12],[15],[3],[10],[14]$} \\
\cline { 2 - 5 } & Deterministic & {$[19]$} & {$[19],[12]$} \\
\cline { 2 - 5 } & Static & & {$[11],[12],[14]$} \\
\cline { 2 - 5 } & Emergency deliveries & {$[11],[15]$} \\
\hline \multirow{3}{*}{ Inventory } & Continuous Review policy & & {$[3],[3]$} \\
\cline { 2 - 5 } & Periodic review policy & & {$[11],[15],[13]$} \\
\cline { 2 - 5 } & Centralized replenishment decisions & {$[19]$} & {$[14],[19]$} \\
\cline { 2 - 5 } & Decentralized replenishment decisions & {$[19]$} & {$[14],[19]$} \\
\hline \multirow{3}{*}{ Product } & Single product & {$[10]$} & {$[3],[10]$} \\
\cline { 2 - 5 } & Multiple products & {$[19]$} & {$[11],[12],[15],[19]$} \\
\hline \multirow{2}{*}{ Number of echelons } & 2 & {$[19]$} & {$[15],[3],[14],[19]$} \\
\cline { 2 - 5 } & 3 & & {$[11],[12]$} \\
\cline { 2 - 5 }
\end{tabular}

involve multiple suppliers and multiple costumers. Multiple multi-echelon inventory models were classified according to a set of criteria. Demand type, review policies, manufacturing single product or multiple products and the number of echelons involved in the system are necessary features and relevant parameters required for formulating and controlling a multi-echelon inventory system. A literature review on the multiechelon pharmaceutical supply chains was elaborated.

Lastly, the literature review on multi-echelon inventory management presented in this paper will be helpful to build alternatives for decision makers in order to choose the appropriate multi-echelon inventory system that corresponds to their priorities and responds to the needs of the whole system. This could be treated in our future work.

This work was conducted within the research project RSCM2015-2018. The authors would like to thank the Moroccan MS, MESRSFC and CNRST for their support.

\section{References}

1. S. Axsäter, Inventory Control (Springer International Publishing, 2015)

2. J.T.Mentzer, W.Dewitt, J.S.Keebler, S.Min, N.W.Nix, C.D.Smith, Z.G.Zacharia. "Defining supply chain management." J. Bus. Logist.,22.2 (2001)

3. R.Ganeshan, "Managing supply chain inventories: a multiple retailer, one warehouse, multiple supplier model.” IJPE, 59 (1999): 341-354

4. P.Vrat, Materials Management (Springer India, 2014)

5. R.Uthayakumar, S.Priyan, "Pharmaceutical supply chain and inventory management strategies for optimization: a study on pharmaceutical company and hospital." ORHC, 2.3 (2013)
6. P.Meindl, S.Chopra, Supply Chain Management, Strategy, Planning, and Operation, (Pearson Education, Inc., 2013).

7. A.J.Clark, "An informal survey of multi-echelon inventory theory.” NRL (1972)

8. S. Axsäter, K.Rosling, "Notes: Installation vs. echelon stock policies for multilevel inventory control.” MS, 39.10 (1993)

9. P.LL, Optimization of $(R, Q)$ policies for multiechelon inventory systems with guaranteed service (LOSI, UTT,2013)

10. H.Scarf, A.J.Clark, "Optimal policies for a multiechelon inventory problem.” MS, 6.4 (1960)

11. W.Zhou, L.Chen, H. Ge, "A multi-product multiechelon inventory control model with joint replenishment strategy.” Appl. Math. Model., 37 (2013): 2039-2050

12. P.Tsiakis, N.Shah, C.C.Pantelides, "Design of multiechelon supply chain networks under demand uncertainty.” Ind. Eng. Chem. Res., 40.16 (2001): 3585-3604

13. E.B.Diks, A.G. de Kok, "Optimal control of a divergent multi-echelon inventory system.” Eur. J. Oper. Res., 111 (1998): 75-97

14. K.Moinzadeh, "A multi-echelon inventory system with information exchange.” MS, 48.3 (2002)

15. W.J.Guerrero, T.G.Yeung, C.Guéret, "Jointoptimization of inventory policies on a multi-product multi-echelon pharmaceutical system with batching and ordering constraints.” Eur. J. Oper. Res., 231 (2013)

16. N.Shah, Comput." Pharmaceutical supply chains: key issues and strategies for optimization." Chem. Eng., 28 (2004): 929-941

17. D.Kim, "An integrated supply chain management system: a case study in healthcare secotr." EC-Web, LNCS, 3590 (2005): 218-227 
18. N.H.Mustaffa, A.Potter, "Healthcare supply chain management in Malaysia: a case study." Supply chain manag. 14. 3 (2009)

19. A.M.M.N.Ahsan, K.A.Zamam, M.N.Sultana, "An optimal joint multi-item replenishment policy for a two level supply chain: centralized versus decentralized decision.” AJOR, 3.2 (2012) 